\title{
Distributed Formation Control of Multiple Unmanned Aerial Vehicles over Time-varying Graphs using Population Games
}

\author{
Julian Barreiro-Gomez, Ignacio Mas, Senior Member, IEEE, Carlos Ocampo-Martinez, Senior Member, IEEE, \\ Ricardo Sanchez-Peña, Senior Member, IEEE, Nicanor Quijano, Senior Member, IEEE
}

\begin{abstract}
This paper presents a control technique based on distributed population dynamics under time-varying communication graphs for a multi-agent system structured in a leaderfollower fashion. Here, the leader agent follows a particular trajectory and the follower agents should track it in a certain organized formation manner. The tracking of the leader can be performed in the position coordinates $x, y$, and $z$, and in the yaw angle $\phi$. Additional features are performed with this method: each agent has only partial knowledge of the position of other agents and not necessarily all agents should communicate to the leader. Moreover, it is possible to integrate a new agent into the formation (or for an agent to leave the formation task) in a dynamical manner. In addition, the formation configuration can be changed along the time, and the distributed populationgames-based controller achieves the new organization goal accommodating conveniently the information-sharing graph in function of the communication range capabilities of each UAV. Finally, several simulations are presented to illustrate different scenarios, e.g., formation with time-varying communication network, and time-varying formation.
\end{abstract}

Index Terms-Unmanned aerial vehicles, distributed formation control, population games, replicator dynamics, timevarying communication network

\section{INTRODUCTION}

The multi unmanned aerial vehicle (UAV) formation has been identified as a cooperative multi-agent control problem of interest because of its complexity, and due to the fact that this type of system can be implemented to perform several tasks and missions, e.g., rescue applications or for military purposes, among others [4]. The multi-agent system formation problem commonly involves spacial constraints. Moreover, UAV technological instruments impose physical limitations such as communication range that constrains the available information that each agent possesses, making their coordinated control challenging. Several control strategies

J. Barreiro-Gomez and C. Ocampo-Martinez are with the Automatic Control Department, Universitat Politècnica de Catalunya, Institut de Robòtica i Informàtica Industrial (CSIC-UPC), Llorens i Artigas, 4-6, 08028 Barcelona, Spain. \{jbarreiro, cocampo\}@iri.upc.edu.

J. Barreiro-Gomez and N. Quijano are with Departamento de Ingeniería Eléctrica y Electrónica, Universidad de los Andes, Carrera $1^{\mathrm{A}}$ No $18 \mathrm{~A}-10$ Bogotá, Colombia \{j.barreiro135, nquijano\}@uniandes.edu.co.

I. Mas and R. Sanchez-Peña are with Instituto Tecnológico de Buenos Aires and Consejo Nacional de Investigaciones Científicas y Técnicas, Ciudad de Buenos Aires C1106ACD, Argentina \{imas, rsanchez\}@itba.edu.ar.

Authors would like to thank Colciencias (grant 6172) and Agència de Gestió d'Ajust Universitaris i de Recerca AGAUR Ref. FI-2014 for supporting J. Barreiro-Gomez. Authors also thank the projects ECOCIS Ref. DPI2013-48243-C2-1-R from the Spanish Ministry of Science and Education, USAITC (RDECOM) with the grant W911NF-14-1-0008, and FONCYT PICT 2014-2055 for partially supporting this research. have been postulated to perform the formation task for multiUAV systems. For instance, a review of different control techniques has been presented in [3], where approaches and issues such as consensus, optimization aspects, distributed tasks assignment and estimation have been discussed. In [8], a cluster-space formulation has been presented for control purposes of multi-agent systems. In [13], formation of UAVs is addressed with optimal control using only information from a fixed neighborhood set. Additionally, [13] discusses the obstacles avoidance issue and the trajectory tracking problem. Although many works consider a distributed communication graph describing information sharing constraints, many of them consider that this network remains constant along the operation of UAVs. This assumption might not be quite realistic, specially when time-varying tasks are taken into account for the UAVs. For instance, in the formation problem, if it is desired that the formation shape varies along the time in a dynamical manner, then the communication capabilities may make necessary to reconfigure the communication network appropriately. In [5] the formation problem of autonomous vehicles is addressed with a receding horizon control approach, and considering time-varying information graphs describing how communication among agents is.

On the other hand, game theory has become a powerful tool for engineering control design. The reason is that game theory allows to model the interaction among different decision makers. Moreover, in [7] a wide review about the role of game theory in the distributed control field is made. Regarding the formation problem, some game-theoretical approaches have been proposed. For instance, a differential game approach is proposed to perform a formation control under distributed fixed communication networks in [6].

Evolutionary game theory, and more specifically population games, have been used as a distributed solution for largescale systems control design. Game-theoretical properties have been used in order to solve distributed constrained optimization problems [2], to perform optimization based on extremum seeking [10], and to solve resource allocation problems [9][11], showing the versatility of this gametheoretical approach. Furthermore, in [1], the distributed population dynamics were presented with their corresponding stability properties. As an alternative in the distributed formation problem of UAVs, this work presents a distributed population-games approach that solves this problem under time-varying communication graphs.

The main contribution of this paper is to exploit the features of distributed population dynamics presented in [1] 
for a multi-agent system formation problem. Additionally to what is discussed in [1], it is pointed out that the stability analysis developed in [1] is extensible to time-varying graphs representing strategy-constrained interactions. In this regard, it is suitable to implement the distributed population dynamics over engineering problems whose communication network varies along the time. In order to illustrate these powerful properties of distributed population dynamics, a distributed formation problem for multi-agent systems under time-varying communication network is proposed. To this end, it is assumed that each agent has a limited communication range, implying that each agent only has partial information about the entire set of agents. Furthermore, it is considered that only few (or only one of the follower agents) are (is) able to communicate to the leader agent that operates as a spacial reference for the whole formation. Additionally, it is shown that an agent can drop or enter from/to the formation without having to modify controllers of the remainder agents. As another relevant feature of the proposed distributed control strategy, the formation shape can be reconfigured dynamically along the time. Finally, simulation results present the behavior of the formation for two different scenarios. First, the case in which a new agent enters the formation dynamically is tested. Secondly, the performance of the proposed distributed controller when the formation is modified along the time is shown.

The remainder of this paper is organized as follows. Section II develops the problem statement corresponding to the multi-agent system to achieve a desired formation.Then, Section III introduces the proposed approach to achieve a formation goal in the leader-follower configuration. Moreover, this section presents preliminary concepts of population games, introduces the distributed replicator dynamics, and describes the general hierarchical control scheme. Afterwards, Section IV exhibits a case study involving six UAVs and simulation results show the formation control performance for two different scenarios. Finally, concluding remarks are drawn and further work is proposed in Section V.

Notation: Column vectors are denoted by bold style, e.g., p. Matrices are denoted by bold upper case, e.g., A. Differently, scalars are denoted by non-bold style, e.g., $n$. The sets are denoted by calligraphic upper case, e.g., $\mathcal{A}$. The diagonal matrix of the vector $\mathbf{x}$ is denoted by $\operatorname{diag}(\mathbf{x})$. Finally, real numbers are denoted by $\mathbb{R}$, and all the nonnegative, and strictly positive real numbers are denoted by $\mathbb{R}_{\geq 0}$, and $\mathbb{R}_{>0}$, respectively. Throughout the paper sub- and super-indices are used. A sub-index refers to a strategy in the game-theoretical context, or to an agent involved in the formation problem in the control context, e.g., in $p_{i}^{d}$, $i$ refers to the $i^{\text {th }}$ strategy or agent. A super-index refers to a population in the game-theoretical context, or to a position parameter $x, y, z$, or $\phi$ in the control context, e.g., in $p_{i}^{d}, d$ refers to the $d^{\text {th }}$ population or position parameter.

\section{PROBLEM STATEMENT OVER INFORMATION GRAPHS}

Consider a set of finite agents (UAVs) denoted by $\mathcal{A}=\{1, \ldots, n\}$, where $n \geq 2$. One of the agents is a leader denoted by $l \in \mathcal{A}$, which influences decisions of the entire set of other agents known as followers, i.e., the set of follower agents is $\mathcal{F}=\mathcal{A} \backslash\{l\}$, and notice that $\mathcal{F} \neq \emptyset$. Each agent $i \in \mathcal{A}$ is located in the positive orthant space, i.e., at non-negative measurable coordinates denoted by $c_{i}^{x}, c_{i}^{y}$, and $c_{i}^{z} \in \mathbb{R}_{\geq 0}$; and with a measurable yaw angle denoted by $c_{i}^{\phi} \in \mathbb{R}_{\geq 0}$ that is considered to be also non-negative. All these measurements are collected in a vector denoted by $\mathbf{c}_{i}=\left[\begin{array}{llll}c_{i}^{x} & c_{i}^{y} & c_{i}^{z} & c_{i}^{\phi}\end{array}\right]^{\top}$, for all $i \in \mathcal{A}$. In addition, let $\mathcal{D}=\{x, y, z, \phi\}$ be the set of all possible position parameters for all the agents from the set $\mathcal{A}$.

On the other hand, it is assumed that each agent can communicate to all other agents, which are located within a communication range radius denoted by $\psi_{i} \in \mathbb{R}_{>0}$, for all $i \in \mathcal{A}$. This situation leads to a time-varying communication network depending on the spacial distribution of agents. This communication network is represented by an undirected graph denoted by $\mathcal{G}(t)=(\mathcal{A}, \mathcal{E}(t), \mathbf{A}(t))$ (the graph is considered to be undirected since each link is assumed to be a bidirectional channel), where $\mathcal{A}$ is the set of nodes corresponding to each agent, $\mathcal{E}(t)$ is the set of links describing the communication network among agents, and $\mathbf{A}(t)=\left[a_{i j}(t)\right]$ is the adjacency matrix whose elements $a_{i j}(t)=1$ if the $i^{\text {th }}$ agent share information with the $j^{\text {th }}$ agent, and $a_{i j}(t)=0$, otherwise. More precisely, the element $a_{i j}(t)$ depends on the communication range given by the $\psi_{i}$, and on the Euclidean distance in $\mathbb{R}^{3}$ as follows: $\xi_{i j}=\sqrt{\left(x_{i}-x_{j}\right)^{2}+\left(y_{i}-y_{j}\right)^{2}+\left(z_{i}-z_{j}\right)^{2}}$, and $a_{i j}(t)=$ $\max \left(0, \operatorname{sgn}\left[\psi_{i}-\xi_{i j}\right]\right)$, where $a_{i j}(t)=a_{j i}(t)$. Then, the constructed adjacency matrix $\mathbf{A}(t)$ implies that each agent $i \in \mathcal{A}$ has a time-varying set of neighbors with whom it can communicate. The set of neighbors for the $i^{\text {th }}$ agent is given by $\mathcal{N}_{i}(t)=\{j:(i, j) \in \mathcal{E}(t)\}$.

Assumption 1: In order to achieve the tracking to the leader satisfying a desired formation shape in a distributed manner, the communication graph should be a connected graph.

The leader agent $l \in \mathcal{A}$ follows a pre-established trajectory, and constitutes a spacial reference for all the follower agents $i \in \mathcal{F}$ to perform a desired formation, i.e., the follower agents $i \in \mathcal{F}$ track the trajectory of the leader $l \in \mathcal{A}$ in an organized manner maintaining a required formation. Furthermore, notice that, depending on the engineering application, the interpretation of the leader might be changed for a target agent, which is wanted to be tracked preserving a formation.

The objective formation is established by assigning desired reference Euclidean distances from each follower $i \in \mathcal{F}$ to the leader $l \in \mathcal{A}$ independently for each coordinate $x, y$, and $z$, and a rotation angle for the yaw $\phi$. These desired distances to achieve the formation are denoted by $r_{i}^{d}$, for all $i \in \mathcal{F}$, and $d \in \mathcal{D}$. Each agent has information about the formation reference distance represented by the vector $\mathbf{r}_{i}=\left[\begin{array}{llll}r_{i}^{x} & r_{i}^{y} & r_{i}^{z} & r_{i}^{\phi}\end{array}\right]^{\top}$, for all $i \in \mathcal{F}$.

For instance, Figure 1(a) shows a diagonal shape whose formation references are given by $\mathbf{r}_{i}=\left[\begin{array}{llll}i & -i & 0 & 0\end{array}\right]^{\top}$, for all $i \in \mathcal{F}$, and Figure 1(b) shows a triangular shape whose 


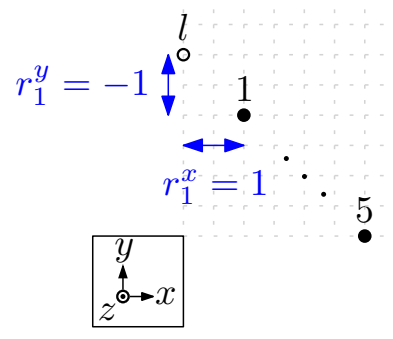

(a)

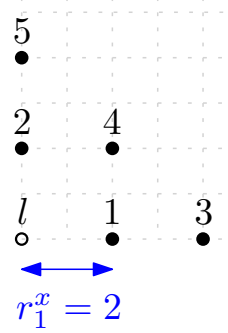

(b)
Fig. 1. Two different possible formations with the same yaw angle $\phi_{i}$, and the same $z_{i}$ coordinate, for all $i \in \mathcal{F}$, an $l \in \mathcal{A}$. (a) diagonal formation, and (b) triangular formation.

formation references are given by $\mathbf{r}_{1}=\left[\begin{array}{llll}2 & 0 & 0 & 0\end{array}\right]^{\top}, \mathbf{r}_{2}=$ $\left[\begin{array}{llll}0 & 2 & 0 & 0\end{array}\right]^{\top}, \mathbf{r}_{3}=\left[\begin{array}{llll}4 & 0 & 0 & 0\end{array}\right]^{\top}, \mathbf{r}_{4}=\left[\begin{array}{llll}2 & 2 & 0 & 0\end{array}\right]^{\top}$, $\mathbf{r}_{5}=\left[\begin{array}{llll}0 & 4 & 0 & 0\end{array}\right]^{\top}$. These formation references play an important role in the design of the distributed populationgames-based formation control presented in Section III.

\section{LEADER-FOLLOWER BASED ON DISTRIBUTED POPULATION GAMES}

Having stated the formation control problem, a distributed strategy based on population games is presented and its main properties and advantages are discussed. Preliminary concepts are introduced, the methodology to address the problem with this approach is presented, and detailed control schemes are explained. Also, an illustrative example for a linear formation is shown for clearness.

\section{A. Background}

Consider four different populations composed of a large and finite number of rational decision makers involved in a strategic interaction. Each population represents a possible position parameter, i.e., the set of populations is given by $\mathcal{D}=\{x, y, z, \phi\}$. These decision makers select among $n$ different available strategies, which represent each one of the agents, i.e., the set of available strategies is given by $\mathcal{A}=\{1, \ldots, n\}$ for all $d \in \mathcal{D}$. Similarly as in Section II, and for notation clearness, $\mathcal{F}=\mathcal{A} \backslash\{l\}$ that is also a subset of strategies, where $l \in \mathcal{A}$ is a fictitious strategy as in [9] corresponding to the leader agent. This mentioned strategy is fictitious due to the fact that there is not a desired position and yaw rotation for the leader agent since it has a preestablished trajectory.

The scalar value $p_{i}^{d} \in \mathbb{R}_{>0}$ is a portion of decision makers selecting the strategy (agent) $i \in \mathcal{F}$ in the population $d \in \mathcal{D}$. Moreover, the portion of decision makers $p_{i}^{d}$ is associated to the corresponding desired position of the $i^{\text {th }}$ agent in the position parameter $d \in \mathcal{D}$. In this regard, notice that the desired position and yaw rotation for the agents is given by $\mathbf{p}_{i}=\left[\begin{array}{llll}p_{i}^{x} & p_{i}^{y} & p_{i}^{x} & p_{i}^{\phi}\end{array}\right]^{\top} \in \mathbb{R}_{>0}^{4}$, for all $i \in \mathcal{F}$. Besides, there is a portion of decision makers selecting the fictitious strategy as in [9] denoted by $p_{l}^{d} \in \mathbb{R}_{\geq 0}$, where $l \in \mathcal{A}, \notin \mathcal{F}$.

On the other hand, $\mathbf{p}^{d}=\left[\begin{array}{lll}p_{1}^{d} & \ldots & p_{n}^{d}\end{array}\right]^{\top} \in \mathbb{R}_{>0}^{n}$ is a vector that collects all the portions of decision makers in the population $d \in \mathcal{D}$. This vector is also known as the population state or the strategic distribution in the respective population. The set of possible strategic distributions for the population $d \in \mathcal{D}$ is given by a simplex defined as follows:

$$
\Delta^{d}=\left\{\mathbf{p}^{d} \in \mathbb{R}_{\geq 0}^{n}: \sum_{i \in \mathcal{A}} p_{i}^{d}=m^{d}\right\},
$$

where $m^{d} \in \mathbb{R}_{>0}$ represents the population size. Furthermore, the population size is associated to the constrained space for the corresponding coordinates $(x, y$, or $z$ ) or rotation $(\phi)$ that agents should respect. Additionally, the interior of the simplex $\Delta^{d}$ is defined as int $\Delta^{d}=\left\{\mathbf{p}^{d} \in \mathbb{R}_{>0}^{n}: \sum_{i \in \mathcal{A}} p_{i}^{d}=m^{d}\right\}$. Notice that the simplex in (1) implies that all the decision makers, corresponding with the follower agents, are constrained to be within a space characterized by

$$
\sum_{i \in \mathcal{F}} p_{i}^{d} \leq m^{d},
$$

which means that it is desired that UAVs positions satisfy a constrained space $\left[\begin{array}{cccc}c_{i}^{x} & c_{i}^{y} & c_{i}^{z} & c_{i}^{\phi}\end{array}\right]^{\top} \leq$ $\left[\begin{array}{llll}m_{i}^{x} & m_{i}^{y} & m_{i}^{z} & m_{i}^{\phi}\end{array}\right]^{\top}$, for all $i \in \mathcal{F}$. Decision makers in the population $d \in \mathcal{D}$ select among the different strategies in order to enhance their benefits. These benefits are described by a function denoted by $f_{i}^{d}$, and whose mapping is $f_{i}^{d}: \Delta^{d} \mapsto \mathbb{R}$, for all $i \in \mathcal{A}$. Besides, let $\mathbf{F}^{d}$ be the vector of fitness functions whose mapping is given by $\mathbf{F}^{d}: \Delta^{d} \mapsto \mathbb{R}^{n}$.

From an evolutionary-game-theoretical perspective, the objective is to achieve convergence to a Nash equilibrium, which is defined next.

Definition 1: (adapted from [12]) $\mathbf{p}^{d *} \in \Delta^{d}$, in the population $d \in \mathcal{D}$, is a Nash equilibrium if each used strategy entails the maximum benefit for the proportion of agents selecting it, i.e., the set of Nash equilibria is given by $\left\{\mathbf{p}^{d *} \in \Delta^{d}: p_{i}^{d *}>0 \Rightarrow f_{i}^{d}\left(\mathbf{p}^{d *}\right) \geq f_{j}^{d}\left(\mathbf{p}^{d *}\right)\right\}$, for all $i, j \in \mathcal{A}$.

Besides, Definition 1 means that if there is not extinction of populations, i.e., $p_{i}^{d}>0$, for all $i \in \mathcal{A}$, then $\mathbf{p}^{*} \in \operatorname{int} \Delta^{d}$, and $f_{i}^{d}\left(\mathbf{p}^{d *}\right)=f_{j}^{d}\left(\mathbf{p}^{d *}\right)$, for all $i, j \in \mathcal{A}$.

Moreover, this work guarantees convergence to the previously defined Nash equilibrium under a class of games known as stable game. This class of games imposes conditions over the fitness functions design as shown in the Definition 2.

Definition 2: (adapted from [12]) The game $\mathbf{F}^{d}$ in the population $d \in \mathcal{D}$ is a stable game if it satisfies the following condition:

$$
\left(\mathbf{p}^{d}-\mathbf{q}^{d}\right)^{\top}\left(\mathbf{F}^{d}\left(\mathbf{p}^{d}\right)-\mathbf{F}^{d}\left(\mathbf{q}^{d}\right)\right) \leq 0,
$$

for all $\mathbf{p}^{d}, \mathbf{q}^{d} \in \Delta^{d}$. Alternatively, condition (3) may be expressed by using the Jacobian matrix of $\mathbf{F}^{d}\left(\mathbf{p}^{d}\right)$, i.e., $D \mathbf{F}^{d}\left(\mathbf{p}^{d}\right)$. If $\mathbf{F}^{d}$ is continuously differentiable, then $\mathbf{F}^{d}$ is stable if and only if $\mathbf{z}^{d^{\top}} D \mathbf{F}^{d}\left(\mathbf{p}^{d}\right) \mathbf{z}^{d} \leq 0$, for all $\mathbf{z}^{d} \in T \Delta^{d}$, and $\mathbf{p}^{d} \in \Delta^{d}$, where $T \Delta^{d}$ is the tangent space of the simplex $\Delta^{d}$, defined by $T \Delta^{d}=\left\{\mathbf{z}^{d} \in \mathbb{R}^{n}: \mathbf{z}^{d^{\top}} \mathbb{1}_{n}=0\right\}$.

There is a relationship between stable games and a type of population games known as full-potential game that is presented in Definition 3. 
Definition 3: (adapted from [12]) If there exists a continuously differentiable function $V\left(\mathbf{p}^{d}\right)$, known as potential function, such that $\mathbf{F}^{d}\left(\mathbf{p}^{d}\right)=\nabla V\left(\mathbf{p}^{d}\right)$, for all $\mathbf{p}^{d} \in \Delta^{d}$, then $\mathbf{F}^{d}$ is a full-potential game.

\section{B. Distributed replicator dynamics and their properties}

The distributed replicator dynamics are one of the fundamental distributed population dynamics presented in [1], which are given by

$$
\dot{p}_{i}^{d}=p_{i}^{d}\left(f_{i}^{d}\left(\mathbf{p}^{d}\right) \sum_{j \in \mathcal{N}_{i}} p_{j}^{d}-\sum_{j \in \mathcal{N}_{i}} p_{j}^{d} f_{j}^{d}\left(\mathbf{p}^{d}\right)\right),
$$

for all $i \in \mathcal{A}$, and $d \in \mathcal{D}$. Alternatively, (4) can be compacted as

$$
\begin{aligned}
\dot{\mathbf{p}}^{d}= & \operatorname{diag}\left(\mathbf{p}^{d}\right) \\
\times & {\left[\operatorname{diag}\left(\mathbf{F}^{d}\left(\mathbf{p}^{d}\right)\right) \mathbf{A} \mathbf{p}^{d}-\mathbf{A d i a g}\left(\mathbf{F}^{d}\left(\mathbf{p}^{d}\right)\right) \mathbf{p}^{d}\right], }
\end{aligned}
$$

for all $d \in \mathcal{D}$, where $\operatorname{diag}\left(\mathbf{p}^{d}\right) \in \mathbb{R}_{\geq 0}^{n \times n}$. The equilibrium points $\mathbf{p}^{*} \in \Delta$ of the distributed replicator dynamics equation (4) implies that the portion of decision makers is $p_{i}=0$ for some $i \in \mathcal{A}$ (there is an extinction for a strategy), or that $f_{i}^{d}\left(\mathbf{p}^{d *}\right)=f_{j}^{d}\left(\mathbf{p}^{d *}\right)$, for all $i, j \in \mathcal{A}, d \in \mathcal{D}$. Consequently, notice that if $\mathbf{p}^{*} \in$ int $\Delta$, and if there is not extinction for any strategy, then the equilibrium point implies that $f_{i}^{d}\left(\mathbf{p}^{d *}\right)=f_{j}^{d}\left(\mathbf{p}^{d *}\right)$, for all $i, j \in \mathcal{A}, d \in \mathcal{D}$.

Then, it is shown that the equilibrium point $\mathbf{p}^{*} \in \operatorname{int} \Delta^{d}$ is asymptotically stable under the distributed replicator dynamics.

Theorem 1: (presented in [1]) Let $F^{d}$ be a full-potential game with strictly concave potential function $f^{d}\left(\mathbf{p}^{d}\right)$, and let $\mathbf{p}^{d *} \in$ int $\Delta^{d}$ be a Nash equilibrium. If the graph $\mathcal{G}=\{\mathcal{A}, \mathcal{E}, \mathbf{A}\}$ is connected and $\mathbf{p}^{d *} \in \Delta^{d}$, then $\mathbf{p}^{d *}$ is asymptotically stable under the distributed replicator dynamics (5).

Proof: The proof for this theorem has been presented in [1] using the following Lyapunov function:

$$
E_{V}\left(\mathbf{p}^{d}\right)=V\left(\mathbf{p}^{d^{*}}\right)-V\left(\mathbf{p}^{d}\right)
$$

where $V\left(\mathbf{p}^{d}\right)$ is the potential function of the potential game $\mathbf{F}^{d}$ (see Definition 3).

In Corollary 1, it is highlighted that Theorem 1 also holds for time-varying communication sharing, broadening the spectrum in engineering applications to implement distributed population games.

Corollary 1: The asymptotic stability of $\mathbf{p}^{d^{*}} \in$ int $\Delta^{d}$ under the distributed replicator dynamics (4) stated in Theorem 1 holds for connected time-varying graphs $\mathcal{G}(t)=(\mathcal{A}, \mathcal{E}(t), \mathbf{A}(t))$, i.e., for time-varying neighborhood $\mathcal{N}_{i}(t)$ for all $i \in \mathcal{A}$. This statement can be seen from Theorem 1 since the Lyapunov function (6) considered in [1] is a common function for all possible connected graph topologies.

Furthermore, the simplex $\Delta^{d}$ is an invariant set under the distributed replicator dynamics (4). This can be seen from the fact that (4) may be re-written in function of the adjacency matrix, i.e.,

$$
\dot{p}_{i}^{d}=\sum_{j \in \mathcal{A}} a_{i j} p_{i}^{d} p_{j}^{d} f_{i}^{d}\left(\mathbf{p}^{d}\right)-\sum_{j \in \mathcal{A}} a_{i j} p_{j}^{d} p_{i}^{d} f_{j}^{d}\left(\mathbf{p}^{d}\right),
$$

for all $i \in \mathcal{A}$, and $d \in \mathcal{D}$. It follows that $\sum_{i \in \mathcal{A}} \dot{p}_{i}^{d}=0$, for all $d \in \mathcal{D}$, due to the fact that $a_{i j}=a_{j i}$. This makes the first and second term in (7) equal showing invariance property of the simplex $\Delta^{d}$, for all $d \in \mathcal{D}$. This property also guarantees that the constraint in (2) is satisfied along the time.

\section{Fitness functions design}

Once the preliminary concepts of population games have been introduced and the distributed replicator dynamics have been presented, the design of the fitness functions is explained and discussed.

The distributed formation problem can be seen as a distributed control in charge of maintaining certain desired distances among a set of agents as presented in Section II (see Figure 1). In this regard, if each agent is controlled in a manner that tracks the leader agent maintaining constant its distance with it, then the formation objective is achieved.

Taking advantage of the asymptotic convergence to a Nash equilibrium $\mathbf{p}^{d^{*}} \in$ int $\Delta^{d}$ under the distributed replicator dynamics, the fitness functions are designed. Let $f_{l}^{d}=-c_{l}^{d}$ be the fitness function assigned to the portion of decision makers selecting the fictitious strategy associated to the leader agent $l \in \mathcal{A}, \notin \mathcal{F}$. Now, let $f_{i}^{d}\left(p_{i}^{d}\right)=r_{i}^{d}-p_{i}^{d}$, for all the portion of decision makers selecting strategies related to follower agents $i \in \mathcal{F}$. First, notice that these fitness functions collected in the vector $\mathbf{F}^{d}\left(\mathbf{p}^{d}\right)$ satisfy the condition for a stable game (see Definition 2). The matrix $D \mathbf{F}^{d}\left(\mathbf{p}^{d}\right)$ is a non-positive diagonal matrix, and with only one null element over the diagonal corresponding to the leader $l \in \mathcal{A}$.

Assuming that there is not extinction of portion of decision makers, it is known that the equilibrium point $\mathbf{p}^{d^{*}} \in$ int $\Delta^{d}$ implies that $f_{i}^{d}\left(\mathbf{p}^{d *}\right)=f_{j}^{d}\left(\mathbf{p}^{d *}\right)$, for all $i, j \in \mathcal{A}, d \in \mathcal{D}$. Then, $f_{l}^{d}\left(\mathbf{p}^{d^{*}}\right)=f_{i}^{d}\left(\mathbf{p}^{d^{*}}\right)$, and $-c_{l}^{d}=r_{i}^{d}-p_{i}^{d^{*}}$, for all $i \in \mathcal{F}, d \in \mathcal{D}$,

$$
p_{i}^{d^{*}}=c_{l}^{d}+r_{i}^{d}, \text { for all } i \in \mathcal{F}, d \in \mathcal{D} .
$$

The equilibrium point condition in (8) shows that all the portion of decision makers for the follower agents $i \in \mathcal{F}$ converges to values such that the formation references are met for all the coordinate positions and yaw angle $d \in \mathcal{D}$ (see Figure 1). Besides, the portion of decision makers $p_{l}^{d}$, for all $d \in \mathcal{D}$, and for the fictitious strategy, adopts a value that satisfies the simplex $\Delta^{d}$.

Remark 1: Notice that it is not necessary that all the follower agents $i \in \mathcal{F}$ have communication to the leader agent $l \in \mathcal{A}, l \notin \mathcal{F}$ (this fact depends on the non-centralized communication topology given by $\mathcal{G}$ ). Nonetheless, all the follower agents -also those without communication to the leader- converge to the appropriate position to achieve the formation with a spacial reference given by the leader agent. 


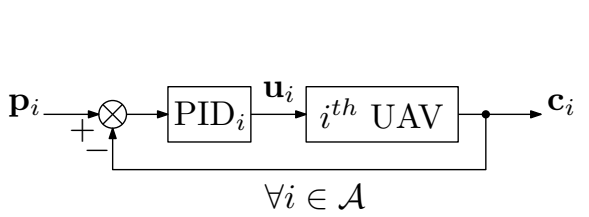

(a)

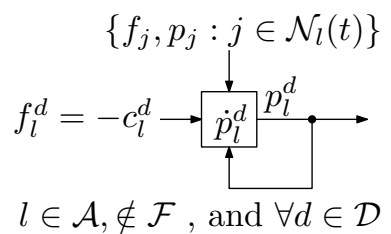

(b)

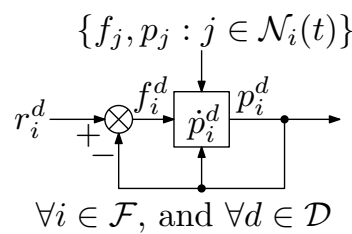

(c)

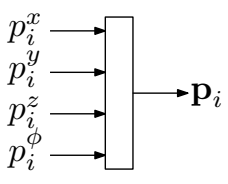

(d)

Fig. 2. Different control stages with their information dependence. (a) Local PID position controller for each agent. It requires information from the distributed population-game-based controller of the strategy $i \in \mathcal{A}$, i.e., $p_{i}^{d}$, for all $d \in \mathcal{D}$. (b) Population-games-based formation control for the fictitious strategy representing the leader agent. It requires information from its neighboors, i.e., $\left\{f_{j}, p_{j}: j \in \mathcal{N}_{l}(t)\right\}$, and the current position of the leader agent $c_{l}^{d}$, for all $d \in \mathcal{D}$. (c) Population-games-based formation control for all the follower agents $i \in \mathcal{F}$. They require information from their neighboors, i.e., $\left\{f_{j}, p_{j}: j \in \mathcal{N}_{i}(t)\right\}$. (d) Collection of all the set-points for the local position control.

\section{Distributed control scheme}

It is assumed that all the follower agents have already a designed local controller in charge of achieving a reference position given by $\mathbf{p}_{i}=\left[\begin{array}{llll}p_{i}^{x} & p_{i}^{y} & p_{i}^{x} & p_{i}^{\phi}\end{array}\right]^{\top} \in \mathbb{R}_{\geq 0}^{4}$ within the positive orthant space. In this paper, these local controllers can be PID as presented in Figure 2(a).

Moreover, depending on the current position of the agents, there is going to be a connected graph $\mathcal{G}(t)$ representing the communication network and possible information sharing. Then, the leader agent disposes of the information given by $\left\{f_{l}, p_{l}\right\} \cup\left\{f_{j}, p_{j}: j \in \mathcal{N}_{l}(t)\right\}$, and the follower agents $i \in \mathcal{F}$ have information given by $\left\{f_{i}, p_{i}\right\} \cup\left\{f_{j}, p_{j}: j \in\right.$ $\left.\mathcal{N}_{i}(t)\right\}$. Figure 2(b) shows the replicator-dynamics-based formation control for the fictitious strategy corresponding to the leader agent $l \in \mathcal{A}$, for all $d \in \mathcal{D}$. Figure 2(c) presents the replicator-dynamics-based formation control for all the follower agents $i \in \mathcal{F}$, and for all $d \in \mathcal{D}$. The formation control in Figure 2(c) generates the appropriate position setpoints, which are collected as presented in Figure 2(d), i.e., $\mathbf{p}_{i}=\left[\begin{array}{llll}p_{i}^{x} & p_{i}^{y} & p_{i}^{x} & p_{i}^{\phi}\end{array}\right]^{\top}$, which are established to the local controllers presented in Figure 2(a). Then, the general control structure composed of local position controllers for each agent, and the distributed formation control, constitutes a hierarchical scheme.

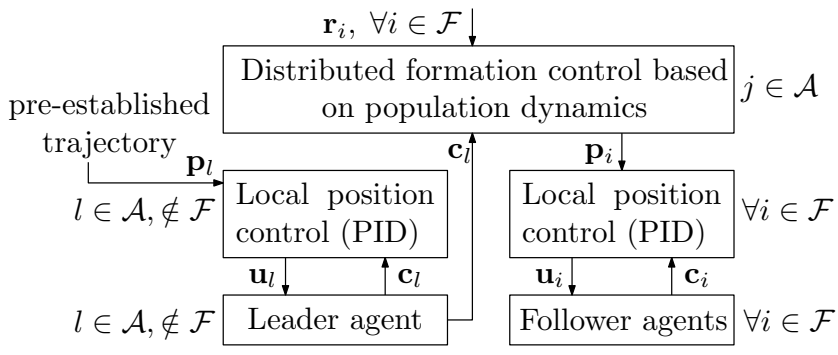

Fig. 3. Hierarchical control scheme for the distributed multi-agent formation.

Figure 3 presents the overall hierarchical control scheme for the distributed replicator-dynamics-based formation controller with time-varying communication network. It can be seen that the leader agent has a local position controller whose reference describes a pre-established trajectory. On the other hand, all the follower agents have a local position controller whose position references $\mathbf{p}_{i}$, for all $i \in \mathcal{F}$, come from the upper layer, which is the distributed replicatordynamics-based formation control. At this upper layer, all the position references $\mathbf{p}_{i}$, for all $i \in \mathcal{F}$, are computed in a distributed manner by using only information about the leader position $\mathbf{c}_{i}$ and the formation references $\mathbf{r}_{i}$, for all $i \in \mathcal{F}$. Notice that the replicator-dynamics-based formation control is distributed since each position reference is computed by using local and partial information.

\section{Simulation ReSUlts}

In order to illustrate the performance of the populationgames approach in the formation control, two different scenarios are considered for $n=6$ agents. For these scenarios, and due to the fact that any agent from the set $\mathcal{A}$ may be the leader, it is selected arbitrarily to be $l=6$. Scenarios are defined as follows:

1) The first scenario consists in a linear formation (see Figure 1(a)). Moreover, a follower agent $5 \in \mathcal{F}$ is initially located out from the communication range of all other agents. The leader trajectory is established in a way that it passes by certain coordinates, such that along the time, the set of agents $\mathcal{A}$ can detect the isolated member including it automatically to the formation. This scenario allows to illustrate the behavior of the distributed game-theoretical approach when a new agent is integrated into the problem.

2) The second scenario consists in a linear formation (see Figure 1(a)) that switches to the triangle formation (see Figure 1(b)) along the time. This scenario allows to show the performance of the distributed game-theoretical approach for time-varying formation objectives.

Figure 4(a) shows the agents trajectory for the linear formation. Figures 4(b)-4(c) show the evolution of the communication network. It can be seen that, at the beginning, the follower agent $5 \in \mathcal{F}$ is isolated and is not able to communicate to any other agent. Consequently, this agent remains without moving in the positive orthant space until time $t=138 \mathrm{~s}$, when it can communicate to agents three and four. Then, agent five is dynamically integrated to the formation problem.

On the other hand, Figure 5(a) shows the agents trajectory for the time-varying formation. First, a linear formation is established and, after this objective is achieved, the objective is changed to a triangular formation at time $t=300 \mathrm{~s}$. Figures 5(b)-5(c) show the evolution of the communication network. It can be seen that, when the formation is varied, the communication network is accommodated conveniently according to the communication range of the agents. 


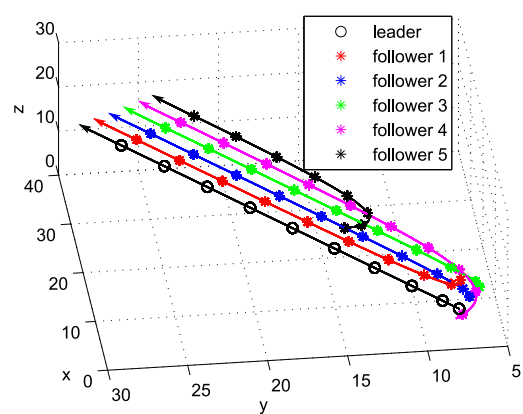

(a)

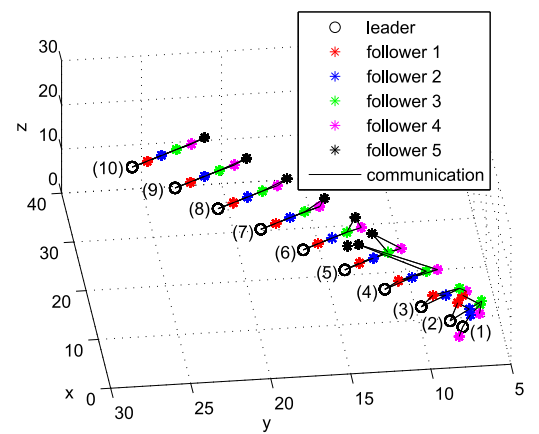

(b)

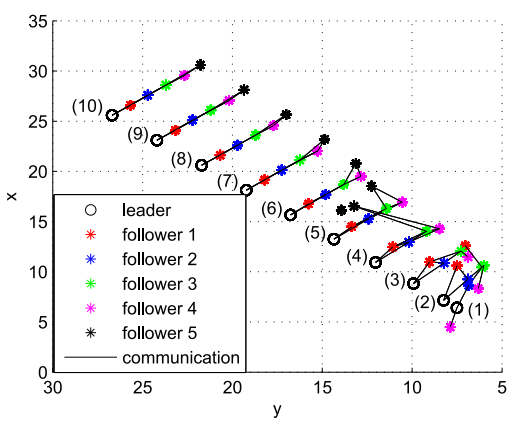

(c)

Fig. 4. Formation result for a line (see Figure 1(a)), range communication $\psi_{i}=3 \mathrm{~m}$, for all $i \in \mathcal{A}$, and illustrating the modularity of the distributed replicator-dynamics-based formation control, i.e., incorporating a new agent to the formation problem along the time. (a) Trajectories of the agents achieving the desired formation. (b)-(c) Evolution of the communication graph for 10 different time instants every 50 seconds, where sequences are as follows: (1) $t=5 \mathrm{~s}$, (2) $t=55 \mathrm{~s}, \ldots$, (10) $t=455 \mathrm{~s}$.

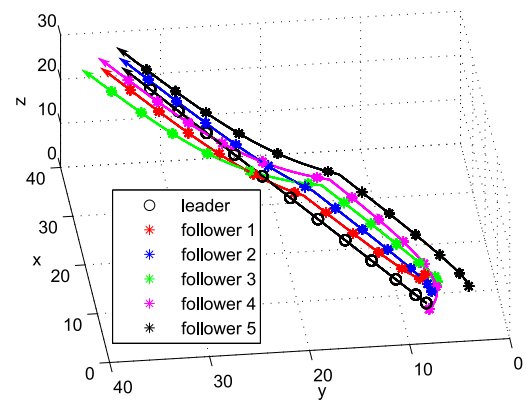

(a)

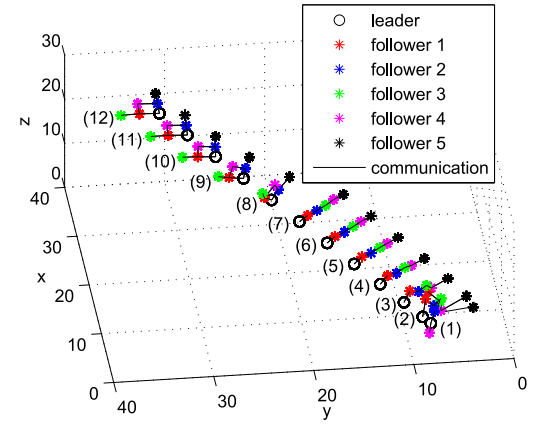

(b)

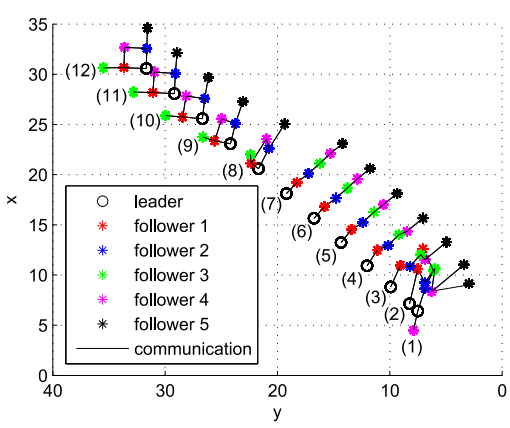

(c)

Fig. 5. Results for changing of topology along the time, from a line (see Figure 1(a)) to a triangle (see Figure 1(b)) with change at $t=300 \mathrm{~s}$, range communication $\psi_{i}=2.5 \mathrm{~m}$, for all $i \in \mathcal{A}$. (a) Trajectories of the agents achieving the desired formation for the line, and then for the triangle. (b)-(c) Evolution of the communication graph for 12 different time instants every 50 seconds, where sequences are as follows: (1) $t=5 \mathrm{~s}$, (2) $t=55 \mathrm{~s}, \ldots$, (12) $t=555 \mathrm{~s}$.

\section{CONCLUSIONS AND FURTHER WORK}

A novel distributed formation control based on population games has been presented. It has been shown that the formation is achieved by using non-centralized communication structures among the agents. In addition, it has been shown that the proposed distributed controller can deal with time-varying formation objectives, and that the informationsharing network can be varied conveniently as a function of the communication range for each agent. Under this timevarying graph scenario, it has been highlighted that stability remains as connectivity of the graph is preserved. Besides, a degree of modularity has been discussed, where a new agent can be incorporated or removed to/from the formation problem in a dynamical manner without affecting the controllers of other agents. As further work, it is proposed to extend the control strategy presented in this paper to consider obstacles located arbitrarily in the positive orthant space including the whole set of agents. Additionally, in order to identify advantages of the proposed formation control, a comparison analysis with other approaches for formation control with time-varying communication graphs is proposed as future work.

\section{REFERENCES}

[1] J Barreiro-Gomez, G Obando, and N Quijano. Distributed population dynamics: Optimization and control applications. IEEE Transactions on Systems, Man, and Cybernetics: Systems, 99:1-11, 2016.
[2] J. Barreiro-Gomez, N. Quijano, and C. Ocampo-Martinez. Constrained distributed optimization: A population dynamics approach. Automatica, 69:101-116, 2016.

[3] Y. Cao, W. Yu, W. Ren, and G. Chen. An overview of recent progress in the study of distributed multi-agent coordination. IEEE Transactions on Industrial Informatics, 9:19-33, 2013.

[4] A. Gautam, P. B. Sujit, and S. Saripalli. A survey of autonomous landing techniques for UAVs. In Proceeding of the 2014 International Conference on Unmanned Aircraft Systems (ICUAS), pages 12101218, 2014.

[5] T. Kevinczky, F. Borrelli, K. Fregene, D. Godbole, and G. Balas. Decentralized receiding horizon control and coordination of autonomous vehicle formation. IEEE Transactions on Control Systems Technology, 16:19-33, 2008.

[6] W. Lin. Distributed UAV formation control using differential game approach. Aerospace Science and Technology, 35:54-62, 2014.

[7] J. Marden and J. Shamma. Game theory and distributed control. Handbook of Game Theory with Economic Applications, 4:861-899, 2015.

[8] I. Mas and C. Kitts. Dynamic control of mobile multirobot systems: the cluster space formulation. IEEE Access, 2:558-570, 2014.

[9] A. Pantoja and N. Quijano. Distributed optimization using population dynamics with a local replicator equation. In Proceedings of the IEEE Conference on Decision and Control (CDC), pages 3790-3795, 2012.

[10] J. Poveda and N. Quijano. Shahshahani gradient-like extremum seeking. Automatica, 58:51-59, 2015.

[11] E. Ramirez-Llanos and N. Quijano. A population dynamics approach for the water distribution problem. International Journal of Control, 83(9):1947-1964, 2010.

[12] W. H. Sandholm. Population games and evolutionary dynamics. Cambridge, Mass. MIT Press, 2010.

[13] J. Wang and M Xin. Integrated optimal formation control of multiple unmanned aerial vehicles. IEEE Transactions on Control Systems Technology, 21:1731-1744, 2012. 